\title{
Crossover between the extended and localized regimes in stochastic partially self-avoiding walks in one-dimensional disordered systems
}

\author{
Juliana Militão Berbert* \\ Instituto de Física Teórica (IFT), Universidade Estadual Paulista (UNESP), Caixa Postal 70532-2, 01156-970 São Paulo, SP, Brazil
}

\author{
Alexandre Souto Martinez ${ }^{\dagger}$ \\ Departamento de Física e Matemática (DFM), Faculdade de Filosofia, Ciências e Letras de Ribeirão Preto (FFCLRP), \\ Universidade de São Paulo (USP) and National Institute of Science and Technology in Complex Systems (LNCT-SC), \\ Avenida Bandeirantes 3900, 14040-901 Ribeirão Preto, SP, Brazil
}

(Received 1 February 2010; revised manuscript received 25 March 2010; published 18 June 2010)

\begin{abstract}
Consider $N$ sites randomly and uniformly distributed in a $d$-dimensional hypercube. A walker explores this disordered medium going to the nearest site, which has not been visited in the last $\mu$ (memory) steps. The walker trajectory is composed of a transient part and a periodic part (cycle). For one-dimensional systems, travelers can or cannot explore all available space, giving rise to a crossover between localized and extended regimes at the critical memory $\mu_{1}=\log _{2} N$. The deterministic rule can be softened to consider more realistic situations with the inclusion of a stochastic parameter $T$ (temperature). In this case, the walker movement is driven by a probability density function parameterized by $T$ and a cost function. The cost function increases as the distance between two sites and favors hops to closer sites. As the temperature increases, the walker can escape from cycles that are reminiscent of the deterministic nature and extend the exploration. Here, we report an analytical model and numerical studies of the influence of the temperature and the critical memory in the exploration of one-dimensional disordered systems.
\end{abstract}

DOI: 10.1103/PhysRevE.81.061127

PACS number(s): 05.40.Fb, 05.60.-k, 05.90.+m, 05.70.Fh

\section{INTRODUCTION}

Stochastic optimization algorithms have been proven to be suitable when difficult (nonpolynomial) problems are addressed. These algorithms have mechanisms of that explore locally and globally the parameter space regions. The local exploration drives the system rapidly to local minima and the global one takes the system away from these minima. Good algorithms are able to effectively combine the amount of local and global exploration. Nevertheless, these algorithms suffer from a priori parametrization such as initial temperature in simulated annealing algorithm [1] or mutation taxes in the genetic algorithm [2]. Further, full system information must be furnished to the algorithm before optimization starts.

Having optimization algorithms in view, here we consider the situation where the information about the system to be optimized is supplied as it evolves. Also, we consider the worst case hypothesis: no correlation in the parameter space. In this case, one can avoid the initial parametrization and the best results are possibly obtained along a line between two regimes: a nonergodic one, which explores locally the parameter space, and the ergodic one, which drives the system away from the local minima.

\section{A. Exploring disordered media: Deterministic and stochastic tourist walks}

Consider a walker exploring a disordered medium with $N$ sites representing, for example, a landscape with feeding

\footnotetext{
*berbertj@gmail.com

†asmartinez@ffclrp.usp.br
}

sites [3], such as flowers for insects, trees for birds, water fountains for animals in general etc. [4-10]. The walker remembers a visited site [11] during its regeneration time (time for it to become attractive to the walker again). After this regeneration time, he/she can, again, revisit this site. If the walker does not know the position of all sites, or, if he/she knows the sites position but have a restrict movement ability, what kind of exploration strategy can emerge from this exploration problem? The tourist walk $[12,13]$ is an exploration strategy that addresses this question, and it can be either deterministic [14] or stochastic [15].

Here, the disordered medium is defined by drawing uniform and randomly each site coordinate along unitary edges of a $d$-dimensional hypercube, $x_{i}^{(k)}, i=1,2, \ldots, N$ and $k$ $=1,2, \ldots, d$. The tourist walk movements are based on the distance between sites. One considers the normalized Euclidean distance between sites, $D_{j, i}=N\left\{\sum_{k=1}^{d}\left[x_{i}^{(k)}-x_{j}^{(k)}\right]^{2}\right\}^{1 / 2}$, to perform the walk. These normalized distances keep the site density constant, for possible comparison between systems with different values of $N$ and/or $d$.

In the deterministic tourist walk (DTW), at each discrete time step, the walker follows the rule of hopping to the nearest site that has not been visited in the last $\mu$ steps. The resulting trajectory is partially self-avoiding, limited by the memory $\mu$. The memory can be understood as the characteristic regeneration time, so that the walker is aware of the $\mu$ nearest neighbors, besides its own position to make movement decisions. Although the rule is simple, nontrivial trajectories emerge from this strategy $[16,17]$. They present a transient part sized $t$, where the walker explores (not necessary) new sites; and a final part, composed by a periodic cycle sized $p \geq \mu+1$, where the walker is trapped in a region visiting only the same sites. The transient time $t$ and the cycle 
period $p$ depends on the medium configuration and the initial condition. A review of the statistical studies of $t$ and $p$ are detailed in Ref. [14]. From those statistical studies, we point out that for $\mu=0$ and 1 , the walker is rapidly trapped in cycles and presents a localized exploration behavior; and for $\mu>1$, the distribution of $p$ presents a whole spectrum of values with possible power-law decay [12,13,16-18]. Applications of these walks are given in Refs. [19-21].

Therefore, to visit all sites, a walker needs a sufficient memory $\mu$ to escape from attractors and extend the exploration. In one-dimensional systems, a crossover between localized (trapping in cycles) and extended (visiting many sites) exploration walker behavior occurs at a critical memory $[16,22]: \mu_{1}=\log _{2} N$. The critical memory is much smaller than the system size and with only local information a walker can present good exploration of the whole system.

The stochastic tourist walk (STW) $[3,23]$ is a generalization of the DTW [12]. In this case, the walker movements are defined by a growing cost function $E\left(D_{j, i}\right)$, by a probability density function (pdf) dependent on the inverse of a formal temperature $T>0$ (the stochastic parameter is $\beta=1 / T$ ) and on the walker memory $\mu \geq 0$. The temperature characterizes the walker exploring ability, namely, the exploration region. For low temperatures, this pdf favors hops to nearest sites. For high temperatures, the walker is able to hop to distant sites. The memory defines an avoidance window with the last $\mu$ visited sites. The pdf, that defines the probability of departing from site $S_{i}$ to site $S_{j}$, is written as: $P_{j, i}^{(\beta, \mu)}$ $=e^{-\beta E\left(D_{j, i}\right)} / Z_{i}^{(\beta, \mu)}$, where $Z_{i}^{(\beta, \mu)}=\sum_{k=1}^{N}{ }^{\prime} e^{-\beta E\left(D_{k, i}\right)}$ is the normalization coefficient. We call attention to the summation $\Sigma^{\prime}$, which excludes the forbidden sites from the avoidance window.

In Ref. [3], the dynamics of STW for walkers, with null memory $(\mu=0)$, exploring disordered $d$-dimensional systems is described. In this case, the order parameter is the mean residence time $\left\langle t_{r}(\beta)\right\rangle$ and for a cost function as $E\left(D_{j, i}\right)=D_{j, i}^{d}$, the $\left\langle t_{r}(\beta)\right\rangle$ and the tail of the nearest distances distribution compete, and a glass transition [24-29] occurs in a finite value: $\beta_{d}=A_{d}$, where $A_{d}$ is the volume of a $d$-dimensional hypersphere with unitary radius. It means that the walker can explore the medium while the stochastic parameter $\beta<A_{d}$. After $\beta=\beta_{d}=A_{d}$ the walker is trapped in regions for long time and the system enters in an out-ofequilibrium regime and an aging effect emerges. For onedimensional systems, $A_{1}=2$, it means that the critical temperature is $T_{0}=1 / 2$. A detailed review is found in Ref. [15].

\section{B. Combining stochasticity and memory}

The results for DTW and STW lead us to believe that the tourist walk problem may present two distinct regimes: a nonergodic one, where the walker is trapped in localized exploration; and an ergodic regime, where the walker can explore the whole medium. We conjecture that to maximize the exploration efficiency it is necessary to choose a cost function that allows a glass transition. Besides, the optimum exploration performance should occur for a specific temperature and memory.
Here, we examine the stochastic tourist walk with memory. We consider the influence of the stochastic parameter and the critical memory in the exploration of onedimensional disordered systems. As discussed below, we have found a transition diagram between localized and extended regimes of exploration. Further, we have also found a relation between the system parameters, which reveals a way to optimize the exploration. The presentation is organized as follow. In Sec. II, in order to find a pdf dependent on both temperature and memory, without restrictions on the summation, we develop an analytical model. In Sec. III, we implement a numerical algorithm based on the developed model. In Sec. IV, the numerical results are discussed. Finally in Sec. V, the final remarks are presented.

\section{ANALYTICAL MODEL}

In this section, we develop an analytical formalism to describe the dynamics of STW when the temperature $T$ and memory $\mu$ vary simultaneously. Our aims are to comprehend the STW dependence on $T$ and $\mu$ and obtain an algorithm for a computational implementation.

The walker movements are defined by a pdf parameterized by a temperature, an avoidance window (characterized by the walker memory) and a cost function (growing on the distances between sites) favoring hops to nearest sites in the low temperature limit. This formalism allows the calculation (at each time interval) of the hopping probability from one site to another of a walker with memory $\mu$ and temperature $T$.

\section{A. Formalism}

Consider $N$ sites drawn by a spatial Poisson distribution along a unitary line segment. Also, consider a walker with memory $\mu$, who has hopped $N_{p}$ sites. First, define two sets: (a) one with all sites: $\mathbb{T}=\left\{S_{1}, S_{2}, \ldots, S_{N}\right\}$, where the indexes label the sites, and (b) other with the $\mu$ forbidden sites forming the avoidance window for step $t: \mathbb{F}(t)$ $=\left\{S_{(t-(\mu-1))}, S_{(t-(\mu-2))}, \ldots, S_{(t)}\right\}$, where the indexes stand for the site position along the walker trajectory. Note that $\mathbb{F}(t) \subset \mathbb{T}$. Second, it is convenient to define another set $\mathbb{K}(t)$, which reveals the correspondence between the indexes of $\mathbb{F}(t)$ and $T$ such that $\mathbb{K}(t)=\left\{k \mid S_{k} \in \mathbb{F}(t)\right\}$. Each site is represented by a unitary value in the state vector of the system:

$$
\left|S_{1}\right\rangle=\left(\begin{array}{c}
1 \\
0 \\
\vdots \\
0
\end{array}\right),\left|S_{2}\right\rangle=\left(\begin{array}{c}
0 \\
1 \\
\vdots \\
0
\end{array}\right), \ldots,\left|S_{N}\right\rangle=\left(\begin{array}{c}
0 \\
0 \\
\vdots \\
1
\end{array}\right) \text {. }
$$

The transposed vectors are: $\left\langle S_{1}\right|=(10 \ldots 0), \ldots, \quad\left\langle S_{N}\right.$ $=(00 \ldots 1)$. It is easy to show the following properties: $\left\langle S_{j} \mid S_{i}\right\rangle=\delta_{j, i}, \sum_{i=1}^{N}\left\langle S_{i} \mid S_{i}\right\rangle=N$ and $\sum_{k=1}^{N}\left|S_{k}\right\rangle\left\langle S_{k}\right|=1$, where 1 is the identity matrix of size $N \times N$.

We also define the operators: memory, cost, density and hopping probability. The memory operator $\mathbb{M}^{(\mu)}(t)=1$ $-\sum_{k \in \mathbb{K}}\left|S_{k}\right\rangle\left\langle S_{k}\right|$ is represented by a diagonal $N \times N$ matrix, with unitary elements, if $S_{j} \notin \mathbb{F}(t) \Rightarrow M_{j, j}^{(\mu)}(t)=1$, otherwise, 


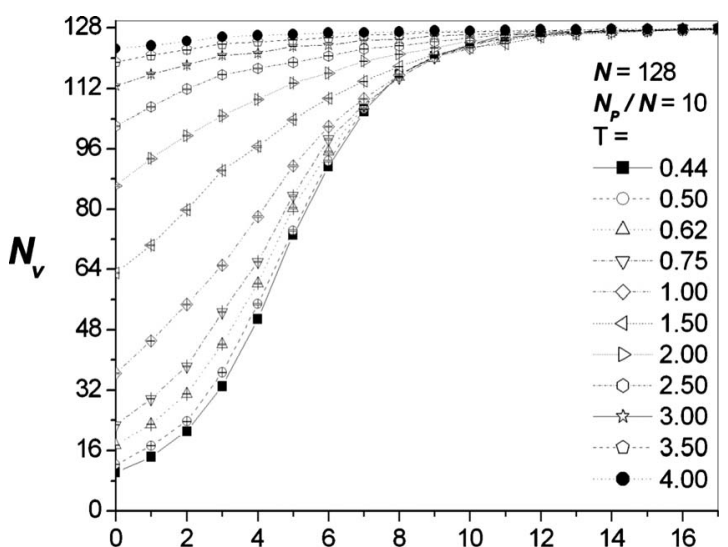

(a)

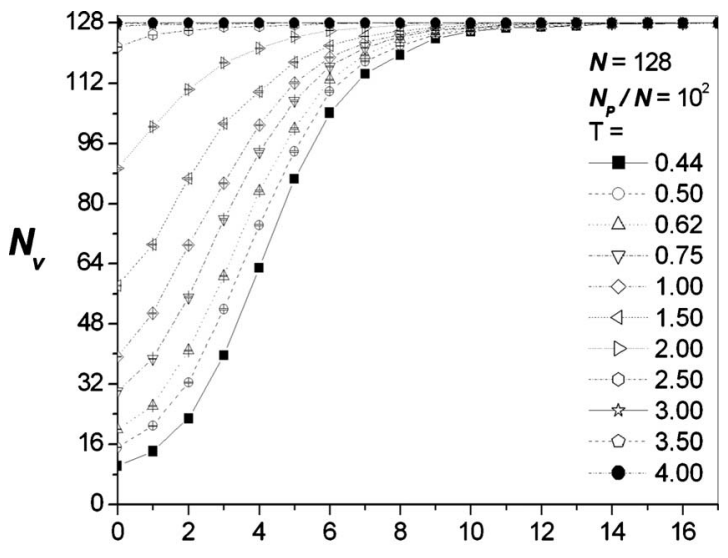

(b)

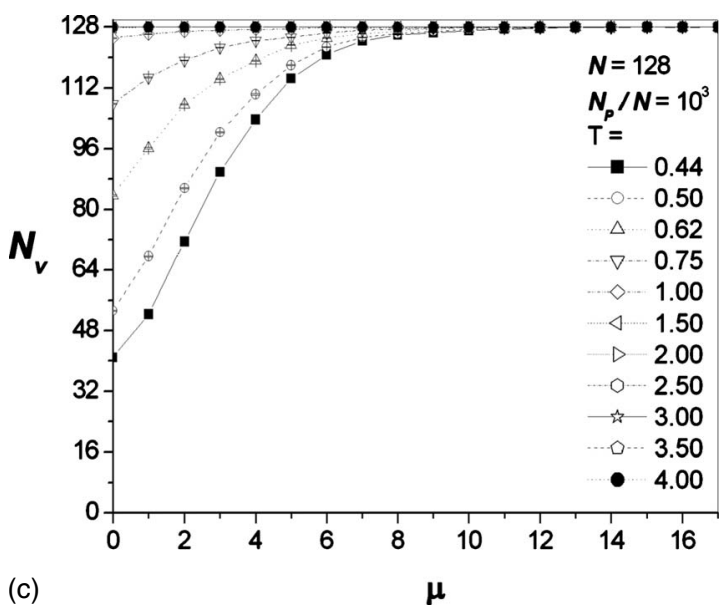

FIG. 1. Number of visited sites $N_{v}$ as a function of walker memory $\mu$ for $N=128$ and different length $N_{p}$ steps: (a) $N_{p} / N=10$, (b) $N_{p} / N=10^{2}$ and (c) $N_{p} / N=10^{3}$. Observe that $N_{v}$ increases as $T$, $\mu$ and $N_{p}$ increase. This dependence suggests that the STW has an aging effect. Numerical data obtained over $10^{3}$ independent trajectories, bars stand for standard error.

elements vanish $\left[S_{j} \in \mathbb{F}(t) \Rightarrow M_{j, j}^{(\mu)}(t)=0\right]$. As the matrix elements are updated, at each step $t$, according to the se $\mathrm{t} \mathbb{F}(t)$ evolution, this operator is time dependent. When this operator is applied to $\left|S_{i}\right\rangle, \mathbb{M}^{(\mu)}(t)$ is updated. After the projection on $\left\langle S_{j}\right|$, only the element $M_{j, j}^{(\mu)}(t)$ is important, since it indicates whether the site $\left\langle S_{j}\right|$ can or cannot be visited. We can express the elements of the memory operator as $\left\langle S_{j}\left|\mathbb{M}^{(\mu)}(t)\right| S_{i}\right\rangle=M_{j, j}^{(\mu)}(t) \delta_{j, i}$, where we emphasize that $M_{j, j}^{(\mu)}(t)$ $=0$, for prohibited sites, or 1 , for allowed ones.

The cost operator is not time dependent. One has $\left\langle S_{j}|\mathbb{H}| S_{i}\right\rangle=E\left(D_{j, i}\right)$, such that $E\left(D_{j, i}\right)$ is a monotonic growing function of the Euclidean normalized distance $D_{j, i}$. This operator is represented as $e^{-\beta \mathrm{H}}$, so that $\left\langle S_{j}\left|e^{-\beta \mathrm{H}}\right| S_{i}\right\rangle=e^{-\beta E\left(D_{j, i}\right)}$

The density operator is defined by $\rho^{(\beta, \mu)}(t)=\mathbb{M}^{(\mu)}(t) e^{-\beta \mathrm{H}}$. It depends on the avoidance window, through $\mathbb{M}^{(\mu)}(t)$, on $\beta$ $=1 / T$ and on the hopping cost function. Using $\left\langle S_{j}\left|\mathbb{M}^{(\mu)}(t)\right| S_{i}\right\rangle$ and $\left\langle S_{j}|\mathbb{H}| S_{i}\right\rangle$, we obtain $\left\langle S_{j}\left|\rho^{(\beta, \mu)}(t)\right| S_{i}\right\rangle=M_{j, j}^{(\mu)}(t) e^{-\beta E\left(D_{j, i}\right)}$.

The normalization coefficient is $Z_{i}^{(\beta, \mu)}(t)$ $=\sum_{k=1}^{N}\left\langle S_{k}\left|\rho^{(\beta, \mu)}(t)\right| S_{i}\right\rangle=\sum_{k=1}^{N} M_{k, k}^{(\mu)}(t) e^{-\beta E\left(D_{k, i}\right)}$. It is important to notice that the summation on $k$ exclude the forbidden sites through the avoidance window with length $\mu$, because $M_{k, k}^{(\mu)}(t)$ is null in this case. This exclusion occurs naturally when we use the operator $\mathbb{M}^{(\mu)}(t)$, avoiding restrictions in the summation.

The probability operator provides the probability of a walker to hop from site $S_{i}$ to $S_{j}$. Therefore, we can define the probability operator: $\quad \mathbb{P}_{i}^{(\beta, \mu)}(t)=\rho^{(\beta, \mu)}(t) / Z_{i}^{(\beta, \mu)}(t)$ $=\mathbb{M}^{(\mu)}(t) e^{-\beta H} / Z_{i}^{(\beta, \mu)}(t)$. Thus, the hopping probability from the site $S_{i}$ to site $S_{j}$ at time step $t$ is:

$$
\left\langle S_{j}\left|\mathbb{P}_{i}^{(\beta, \mu)}(t)\right| S_{i}\right\rangle=\frac{M_{j, j}^{(\mu)}(t) e^{-\beta E\left(D_{j, i}\right)}}{\sum_{k=1}^{N} M_{k, k}^{(\mu)}(t) e^{-\beta E\left(D_{k, i}\right)}}=P_{j, i}^{(\beta, \mu)}(t) .
$$

Notice two properties of this operator: $\sum_{i=1}^{N}\left\langle S_{j}\left|\mathbb{P}_{i}^{(\beta, \mu)}(t)\right| S_{i}\right\rangle$ $=1$ and $\sum_{i, j=1}^{N}\left\langle S_{j}\left|\mathbb{P}_{i}^{(\beta, \mu)}(t)\right| S_{i}\right\rangle=N$. It is worth noting that Eq. (1) binds $T$ and $\mu$ in the same expression, without restrictions. For $\mu=0$, this definition allows the walker to remain in the same site $S_{j}=S_{i}$. In this case, the probability does not vanish only if $M_{i, i}^{(\mu)}(t) \neq 0$, which occurs for $\mu=0$.

\section{B. Hopping probability}

Consider just one trajectory with length $N_{p}$ hops (steps), connecting the initial and final sites. The probability of a walker, departing from $S_{i}$ and arriving at $S_{j}$ passing through fixed sites $S_{k_{a}}, a=1,2, \ldots,\left(N_{p}-1\right)$, is written as

$$
\widetilde{P}_{S_{j} \leftarrow S_{i}}^{\left(N_{p}\right)}=P_{j, k_{N_{p}-1}}^{(\beta, \mu)}\left(N_{p}\right)\left(\prod_{r=1}^{N_{p}-2} P_{k_{r+1}, k_{r}}^{(\beta, \mu)}(r+1)\right) P_{k_{1}, i}^{(\beta, \mu)}(1) .
$$

Notice that, due to the walker memory, this probability vanishes if one of the sites is prohibited to be visited. Therefore, for different $\mu$ values, the walker finds different restrictions about the following site to be visited.

In the high temperature limit $(\beta \rightarrow 0)$, the cost operator $e^{-\beta H}$ dependence on the distance between sites is negligible. Thus, the hopping probability depends only on the walker memory. Using Eq. (2), the hopping probability between sites $S_{i}$ and $S_{j}$, passing through $N_{p}-1$ nonforbidden intermediary sites [just when $M_{k, k}^{(\mu)}(t)=1$ ] is $\widetilde{P}_{S_{j} \leftarrow S_{i}}^{\left(N_{p}\right)}=(N-\mu)^{-N_{p}}$, where all sites, which do not belong to the avoidance window, have the same probability to be visited. In the particular case $\mu=0\left(\widetilde{P}_{S_{j} \leftarrow S_{i}}^{\left(N_{p}\right)}{ }=N^{-N_{p}}\right)$, the system is ergodic, since the 
walker can be found at any site (the system can be found in any of its states) with the same probability. Further, in this case, the system is in equilibrium, since the detailed balance is satisfied on the master equation, $P_{j, i}^{(\beta, 0)}=P_{i, j}^{(\beta, 0)}$, independently of the number of steps between $S_{i}$ and $S_{j}$. For the low temperature limit $(\beta \gg 1)$, the exponential factor of the Eq. (1), is dominated, at each step, by the hop to the nearest allowed site, and the DTW is recovered.

\section{COMPUTATIONAL IMPLEMENTATION}

To understand the microscopic properties of the STW, we have implemented a numerical simulation code using the Monte Carlo method for STW in one-dimensional systems. The developed algorithm is based on the analytical development (Sec. II) and it is composed, essentially, by three parts: (i) the map generation module sets up the disordered medium, composed by $N$ sites distributed by a Poisson process, and each map generates a distance matrix; (ii) the calculating probability module, first, calculates the exponentials of the Eq. (1), using $E\left(D_{j, i}\right)=D_{j, i}$; then it calculates the probability at each step and (iii) the STW module account for the walker decision, calculating $P_{j, i}^{(\beta, \mu)}$, and following the rule of not returning to the last $\mu$ visited sites, for $N_{p}$ steps.

To understand the STW dynamics, we have calculated the density $\rho=N_{v} / N$ of distinctly visited sites $N_{v}$, for several trajectories over several maps. The quantity $\rho$ is the order parameter. In this way, we aspire to find a relation between $T, \mu$ and $N_{p}$ that allows the optimization of the walker exploration.

We have validated our algorithm throughout the analytical considerations in the high and low temperature limits (Sec. II). We have calculated some probabilities for the high temperature limit $(\beta \rightarrow 0)$, for different values of the walker memory and found that the hopping probability at each step is $P_{j, i}^{(\beta=0.1, \mu)} \approx 1 /(N-\mu)$; after $N_{p}$ steps, the probability of the trajectory was $\widetilde{P}_{S_{j}}^{\left(N_{p}\right)} S_{i} \approx[1 /(N-\mu)]^{N_{p}}$. For low temperature limit, we have observed that the walker hops to the nearest allowed neighbors. With the validated algorithm, we have calculated $\rho$ for $10^{3}$ independent trajectories. We have considered the temperature interval $T=[0.1 ; 4]$, walker memory interval $\mu=[0,20]$, number of sites $N=\{64,128\}$ and number of steps $N_{p}=\left\{10,10^{2}, 10^{3}, 4000\right\} N$.

\section{NUMERICAL RESULTS}

Here, we report the numerical results for STW in onedimensional systems. From each trajectory, we have obtained the density of distinctly visited sites $\rho$, for different values of $T, \mu$, and $N_{p}$. In Fig. 1 , we show how the number of distinctly visited sites $N_{v}$ increases as $T, \mu$, and $N_{p}$ increase. The exploration dependence on $N_{p}$ suggests that STW has an aging effect. For specific values of $T$ and $N_{p}, N_{v}$ shows a crossover between the localized (few visited sites) and extended (many visited sites) exploration regimes. This kind of crossover is also found in the deterministic tourist walk [14].

The $\rho(\mu)$ inflection point (Fig. 2) gives us the critical memory $\mu_{1}$, where the mentioned crossover occurs. To determine this point we have fitted data with,

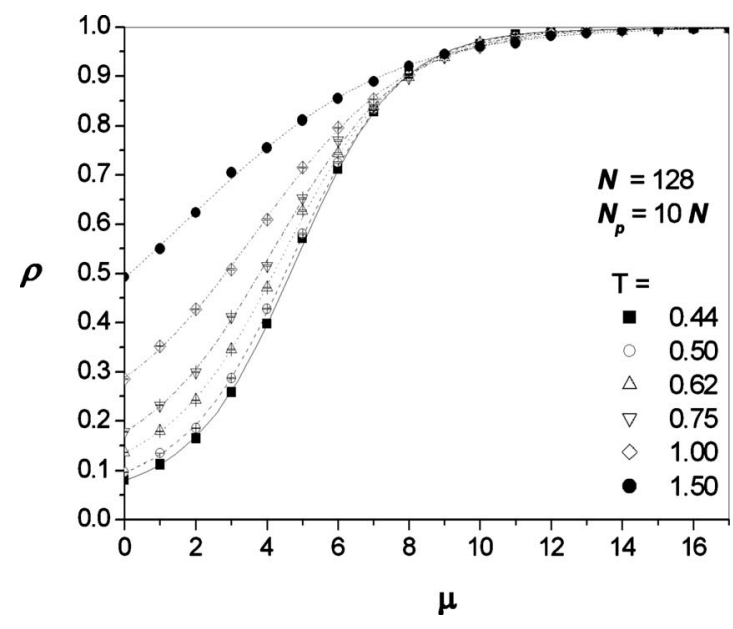

FIG. 2. Density of visited sites $\rho$ as a function of the walker memory $\mu$ for maps with $N=128$ and with $N_{p}=10 N$ steps, for temperature values $T=0.44 ; 0.50 ; 0.62 ; 0.75 ; 1.0$ and 1.5 . The symbols stand for numerical data, and lines are the curves fitted by Eq. (3). From these fits one obtains the critical memory $\mu_{1}$. The numerical data have been obtained with $10^{3}$ independent trajectories, bars stand for standard error.

$$
\rho=\frac{\rho_{\min }-\rho_{\max }}{1+\exp \left[k\left(\mu-\mu_{1}\right)\right]}+\rho_{\max },
$$

where $k$ is a scale factor, $\rho_{\min }$ and $\rho_{\max }$ are the $\rho$ maximum and minimum values, respectively. In the numerical experiments, $\rho_{\max }=1$, namely, after certain value of memory, the walker visits all sites, at least once. We have tried to fit data with other models, such as the hyperbolic tangent, however, Eq. (3) gives better results.

We have plotted $T\left(\mu_{1}\right)$, for maps with $N=128$ sites and $N_{p} / N=10,10^{2}$ and $10^{3}$, and found a linear behavior as shown in Fig. 3. Besides, as $N_{p}$ increases, the curve $T\left(\mu_{1}\right)$ approaches to the curve given by Eq. (4),

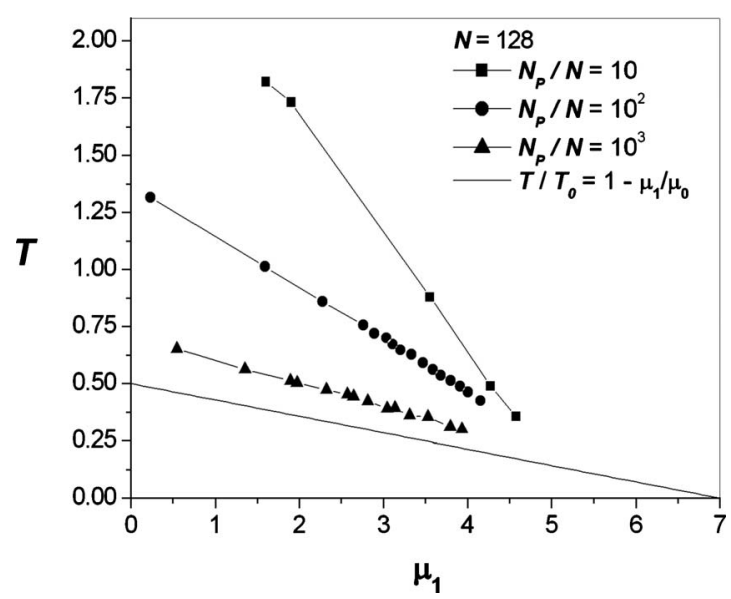

FIG. 3. These plots suggest that as $N_{p}$ increases, $T\left(\mu_{1}\right)$ approaches the linear behavior of Eq. (4). This equation connects the purely deterministic $\left(T=0 \Rightarrow \mu_{1}=7\right)$ and purely stochastic $(\mu=0 \Rightarrow T=1 / 2)$ cases. 


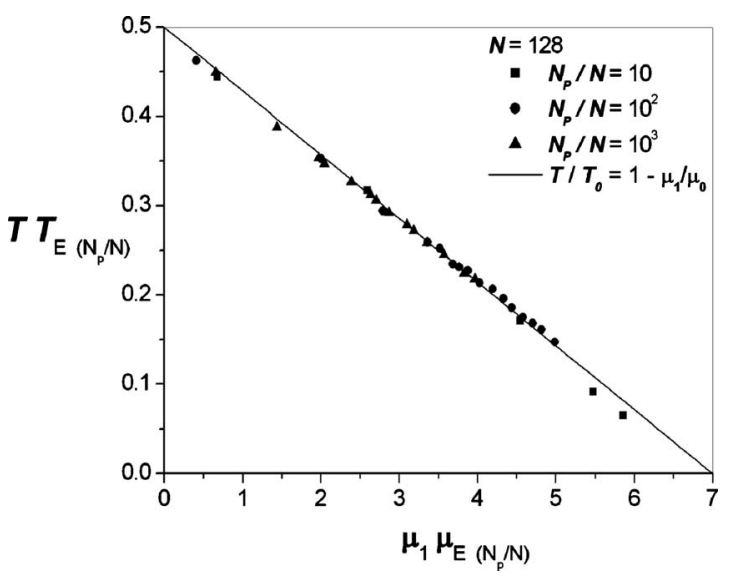

FIG. 4. We have found a data collapse simply by multiplying the temperature by $T_{E}$ and the memory by $\mu_{E}$. The scale factors $T_{E}$ and $\mu_{E}$ depend on $N_{p} / N$, as one can see in Fig. 5. The data collapse reveals that it is possible to find a relation between $T, \mu, N$, and $N_{p}$.

$$
\frac{T}{T_{0}}=1-\frac{\mu_{1}}{\mu_{0}},
$$

where $T_{0}$ is the transition temperature when $\mu=0$, and $\mu_{0}$ is the critical memory when $T=0$. This equation just connects the purely determinist case $(T=0$ and the critical memory is $\mu_{0}=\log _{2} N$, for maps with $N=128, \mu_{0}=7$ ) and purely stochastic case $\left(\mu=0\right.$, and a glass transition at temperature $T_{0}$ $=1 / 2$ ). The connection represented by Eq. (4) also corresponds to the hypothesis that as the walker exploration time $N_{p}$ increases, the curve connecting the extreme cases of the tourist walk, $\left(0, T_{0}\right)$ and $\left(\mu_{0}, 0\right)$, is achieved.

To corroborate our hypothesis, we have looked for a general expression, that (i) depends on $N_{p}$, (ii) fits the numerical data and, (iii) when extrapolated to the maximum $N_{p}$ leads to Eq. (4). For each value of $N_{p}$, we have found scale factors $T_{E}$ and $\mu_{E}$ to multiply $T$ and $\mu_{1}$, respectively. This procedure give us a data collapse (see Fig. 4) for three different $N_{p}$ values. In this way, one obtains a crossover diagram between localized and extended exploration regimes governed by a general expression as function of $T, \mu, N$, and $N_{p}$. Plotting $T_{E}$ and $\mu_{E}$ as function of $N_{p} / N$ (Fig. 5), we obtain their dependence on the ratio $\left(N_{p} / N\right)$,

$$
T_{E}=\frac{1}{10}\left(\frac{N_{p}}{N}\right)^{5 \gamma} \text { and } \mu_{E}=\frac{3}{2}\left(\frac{N_{p}}{N}\right)^{-\gamma} \text {, }
$$

where $\gamma=1 / 18$. Notice that $T_{E} \propto \mu_{E}^{-5}$. Jointing Eqs. (4) and (5), we obtain

$$
\frac{T}{T_{0}} T_{E}=1-\frac{\mu_{1}}{\mu_{0}} \mu_{E}
$$

and we are able to discriminate the localized and extended exploration regimes as function of $T, \mu, N$ and $N_{p}$,

$$
\frac{1}{10} \frac{T}{T_{0}}\left(\frac{N_{p}}{N}\right)^{5 / 18}=1-\frac{3}{2} \frac{\mu_{1}}{\mu_{0}}\left(\frac{N_{p}}{N}\right)^{-1 / 18} .
$$

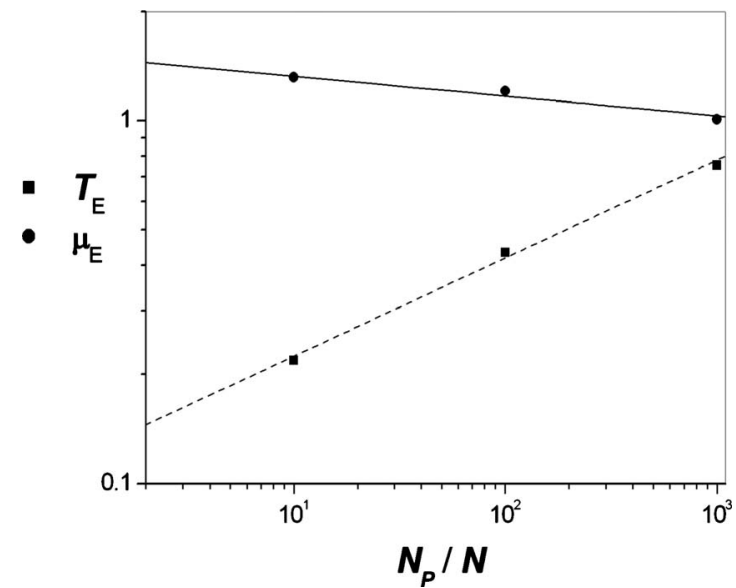

FIG. 5. The scale factors $T_{E}$ (square) and $\mu_{E}$ (circles) as a function of $N_{p} / N$ in log-log scale. The fits lead to $T_{E}$ (dash-line) and $\mu_{E}$ (continuous-line) of Eq. (5).

Moreover, using $T_{E}=1$ (or $\mu_{E}=1$ ), one finds the ratio $N_{p} / N$ that provides the hypothesis in Eq. (4). Thus: $T_{E}=\left(N_{p} / N\right)^{5 \gamma / 10}=1,\left(N_{p} / N\right)^{5 \gamma}=10$ and $N_{p} / N=10^{1 / 5 \gamma}$,

$$
\left(\frac{N_{p}}{N}\right)_{\max } \approx 4000 \text {. }
$$

In this way, Eq. (7) (i) gives a transition diagram between localized and extended exploration regimes, and (ii) fits the numerical data. Its resolution is meaningful for a walker exploring a disordered medium with $N$ sites for $N_{p}=4000 N$ steps (exploration time).

We have also performed the STW over a medium with $N=64$ sites and let the walker to explore it for $N_{p} / N$ $=10,10^{2}, 10^{3}$, and 4000 steps. Again, the numerical data were well fitted by Eq. (7) (see Fig. 6). Moreover, for $N_{p} / N=4000$ steps, the asymptotic values of Eq. (4) are reached.

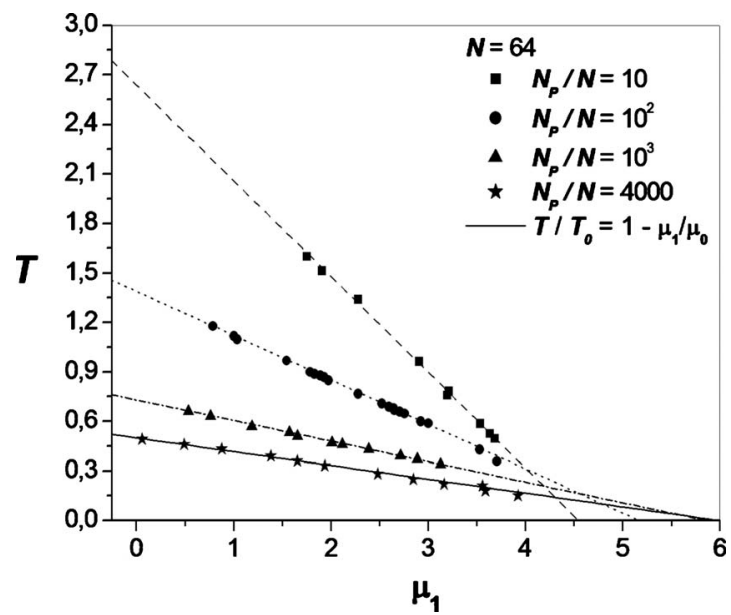

FIG. 6. The dependence of temperature $T$ as a function of $\mu_{1}$, for $N=64$. The symbols stand for numerical results and the segmented lines have been calculated by Eq. (7), for $N_{p} / N=10$ (dash), $N_{p} / N=10^{2}$ (dot) and $N_{p} / N=10^{3}$ (dash-dot). The continuous line corresponds to our hypothesis of $N_{p} / N=4000$ in Eq. (7). 


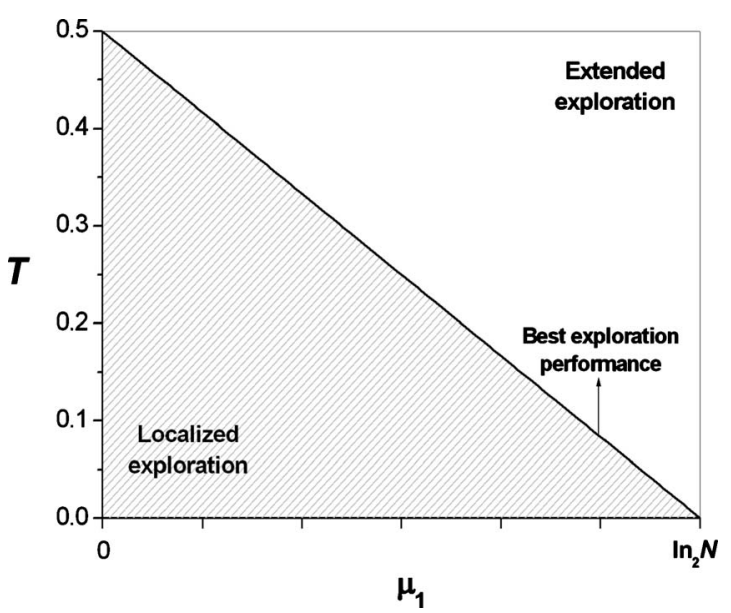

FIG. 7. Transition diagram for an arbitrary value of $N$. The line is given by Eq. (7). It is along this line that the best medium exploration performance is achieved. The lower region corresponds to the localized exploration regime and the upper one corresponds to the extended exploration regime.

The maximum exploration time $N_{p}=4000 \mathrm{~N}$ is an optimization time for the walker exploration. In other words, this is the minimum time that allows a walker, with short memory, to visit all sites, at least once, spending minimum energy $\left(E\left(D_{j, i}\right)\right)$. In this way, a walker, with temperature $T$ and memory $\mu_{1}$ who explores a medium of size $N$ for $N_{p}$ steps, has its optimum performance when $T, \mu_{1}, N$, and $N_{p}$ are related by Eq. (7) and the maximum exploration time is given by Eq. (8).

Therefore, the main numerical results that can be pointed out are: (i) numerical data collapse, that leads to a universality in out-of-equilibrium systems, as the STW problem; (ii) correction factor for the finite-size effect (dependence on $N$ ) and aging effect (dependence on $N_{p}$ ); (iii) transition diagram (Fig. 7) between localized and extended exploration regimes and (iv) relation between $T, \mu_{1}, N$, and $N_{p}$, that reveals a way to optimize the exploration of a disordered medium.

\section{CONCLUSIONS}

We have investigated the stochastic tourist walk in disordered one-dimensional systems, varying simultaneously the medium temperature $T$ and walker memory $\mu$. An analytical formalism has been developed to obtain a hopping probability as function of $T$ and $\mu$, following the rule of not returning to the last $\mu$ visited sites. The order parameter is the density of distinctly visited sites $\rho$. This quantity has allowed us to relate the quantities $T, \mu$, and $N_{p}$ throughout a numerical data collapse. This collapse implies in universality. Thus, the stochastic tourist walk is an example that systems out of thermodynamic equilibrium can present universality. The relationship between $T, \mu$, and $N_{p}$ [Eq. (7)] assures an optimal walker medium exploration. Exploration time is given by Eq. (8). Finally, the fundamental result is the transition line, which discriminates the localized and extended exploration regions. In these regions, a walker explores few (localized) or many (extended) sites, performing the partially selfavoiding stochastic tourist walk. We believe that a consistent choice of values for temperature and memory (along the transition line) gives the best compromise between maximum exploration and minimum trajectory (exploration time). For this reason, a thorough analysis of the STW problem in higher dimensionalities is interesting to develop an optimization algorithm.

\section{ACKNOWLEDGMENTS}

The authors thank Rodrigo Silva González, Marco Antonio Alves da Silva, and Roberto Andre Kraenkel for useful comments and nice discussion. A.S.M. acknowledges the support of the Brazilian agency CNPq (Grants No. 303990/ 2007-4 and No. 476862/2007-8). J.M.B. acknowledges the support of the Brazilian agencies CNPq (Grant No. 134461/ 2007-0) and FAPESP (Grant No. 2009/11567-6).
[1] S. Kirkpatrick, C. D. Gelatt, and M. P. Vecchi, Science 220, 671 (1983).

[2] D. E. Goldberg, Genetic Algorithms (Addison-Wesley, Reading, MA, 1989).

[3] A. S. Martinez, O. Kinouchi, and S. Risau-Gusman, Phys. Rev. E 69, 017101 (2004).

[4] G. M. Viswanathan, V. Afanasyev, S. Buldyrev, E. J. Murphy, P. A. Prince, and H. E. Stanley, Nature (London) 381, 413 (1996).

[5] G. M. Viswanathan, S. Buldyrev, S. Havlin, M. G. E. da Luz, E. P. Raposol, and H. E. Stanley, Nature (London) 401, 911 (1999).

[6] D. Boyer, O. Miramontes, G. Ramos-Fernandez, J. L. Mateos, and G. Cocho, Physica A 342, 329 (2004).

[7] D. Boyer and H. Larralde, Complexity 10, 52 (2005).

[8] D. Boyer, G. Ramos-Fernandez, O. M. J. L. Mateos, G. Cocho,
H. Larralde, H. Ramos, and F. Rojas, Proc. Biol. Sci. 273, 1743 (2006).

[9] M. C. Santos, D. Boyer, O. Miramontes, G. M. Viswanathan, E. P. Raposo, J. L. Mateos, and M. G. E. da Luz, Phys. Rev. E 75, 061114 (2007).

[10] A. M. Edwards, R. A. Phillips, N. W. Watkins, M. P. Freeman, E. J. Murphy, V. Afanasyev, S. V. Buldyrev, M. G. E. da Luz, E. P. Raposo, and H. E. Stanley, Nature (London) 449, 1044 (2007).

[11] J. C. Cressoni, Marco Antonio Alves da Silva, and G. M. Viswanathan, Phys. Rev. Lett. 98, 070603 (2007).

[12] G. F. Lima, A. S. Martinez, and O. Kinouchi, Phys. Rev. Lett. 87, 010603 (2001).

[13] H. E. Stanley and S. V. Buldyrev, Nature (London) 413, 373 (2001).

[14] R. S. González, J. M. Berbert, C. A. S. Terçariol, W. T. R. 
Oliveira, and A. S. Martinez, J. Comput. Interdisciplinary Sci. 1 (2), 119 (2009).

[15] J. M. Berbert, R. S. González, and A. S. Martinez, J. Comput. Interdisciplinary Sci. 1 (1), 33 (2008).

[16] C. A. S. Terçariol, R. S. González, and A. S. Martinez, Phys. Rev. E 75, 061117 (2007).

[17] C. A. S. Terçariol and A. S. Martinez, Phys. Rev. E 78, 031111 (2008).

[18] O. Kinouchi, A. S. Martinez, G. F. Lima, G. M. Lourenço, and S. Risau-Gusman, Physica A 315, 665 (2002).

[19] M. G. Campiteli, P. D. Batista, O. Kinouchi, and A. S. Martinez, Phys. Rev. E 74, 026703 (2006).

[20] A. R. Backes, W. N. Gonçalves, A. S. Martinez, and O. M. Bruno, Pattern Recogn. 43, 685 (2010).

[21] A. R. Backes, A. S. Martinez, and O. M. Bruno, Pattern Recogn. Lett. (to be published).
[22] C. A. S. Terçariol, R. S. González, W. T. R. Oliveira, and A. S. Martinez, Physica A 386, 678 (2007).

[23] S. Risau-Gusman, A. S. Martinez, and O. Kinouchi, Phys. Rev. E 68, 016104 (2003).

[24] J.-P. Bouchaud, J. Phys. I 2, 1705 (1992).

[25] J. C. Dyre, Phys. Rev. B 51, 12276 (1995).

[26] C. Monthus and J.-P. Bouchaud, J. Phys. A 29, 3847 (1996).

[27] B. Rinn, P. Maass, and J.-P. Bouchaud, Phys. Rev. B 64, 104417 (2001).

[28] E. M. Bertin and J. P. Bouchaud, Phys. Rev. E 67, 026128 (2003).

[29] R. A. Denny, D. R. Reichman, and J.-P. Bouchaud, Phys. Rev. Lett. 90, 025503 (2003). 